\title{
Genetic Algorithm Aided Transmit Power Control in Cognitive Radio Networks
}

\author{
Raikel B. López*, Samuel M. Sánchez*, Evelio M. G. Fernandez ${ }^{\dagger}$, Richard D. Souza ${ }^{\ddagger}$ and Hirley Alves ${ }^{\S}$ \\ *Central University of Las Villas, Santa Clara, Cuba - \{rbardon, montejo\}@uclv.edu.cu \\ ${ }^{\dagger}$ Federal University of Paraná, Curitiba, Brazil - evelio@eletrica.ufpr.br \\ ${ }_{\ddagger}^{\ddagger}$ Federal University of Technology - Paraná (UTFPR), Curitiba, Brazil - richard@utfpr.edu.br

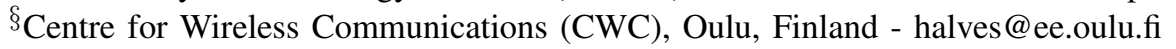

\begin{abstract}
We address the power control problem in cognitive radio networks where secondary users exploit spatial spectrum opportunities without causing unacceptable interference to primary users. An optimization problem is formulated aiming at maximizing the utility of secondary users and to ensure the QoS for both primary and secondary users. To solve the power allocation problem a genetic algorithm is developed, and two fitness functions are proposed. The first is oriented towards minimizing the total transmit power consumption of the secondary network. The second is a multi-objective function and is oriented to the joint optimization of total capacity and transmit power consumption of the secondary network. Results show a near-optimum performance of the genetic algorithm aided power control scheme based on the multi-objective fitness function.
\end{abstract}

\section{INTRODUCTION}

Cognitive radio technology can significantly improve spectrum utilization efficiency by allowing secondary users (SUs) to dynamically access and share licensed frequency bands which are assigned to primary users (PUs) [1]. Two main paradigms have been proposed for spectrum access in cognitive radio networks, namely, the opportunistic spectrum access (OSA) and the concurrent spectrum access (CSA) models [2]. OSA systems aim at avoiding concurrent transmissions between PUs and SUs by restricting the SUs to access the channel only at temporary unused frequency bands. The CSA model is based on the idea that SUs are allowed to transmit in the same frequency used by active PUs, but subject to the constraint that they must not affect the communications of PUs.

Transmit power control (TPC) techniques at the SUs may enable CSA without causing harmful interference to PUs. The use of TPC may also provide benefits in terms of energy consumption [3], [4] and network throughput [5]-[7]. The TPC algorithm can be either distributed or centralized. In distributed algorithms, every node individually adjusts its transmit power in order to maximize its own utility function. In centralized algorithms the TPC problem is addressed as a global optimization problem, where the goal is to maximize a unique utility function for the secondary network.

In [3] a distributed TPC algorithm based on noncooperative game theory for energy efficiency maximization in the uplink of a multiuser cognitive radio network is proposed. The tradeoff between energy consumption minimization and throughput maximization for the secondary link based on TPC strategies is discussed in [4]. In [5] a robust TPC problem is formulated as a global optimization taking into account the uncertainties on the channel estimation, employing a Lagrangian dual function to solve the problem in a distributed way. In [6] the joint design of antenna beamforming and power allocation in cellular cognitive radio networks is considered and a centralized algorithm to maximize the secondary network sum rate is proposed. In [7] a TPC algorithm is proposed in which cognitive nodes exploit location awareness to find the optimal power control by an exhaustive search technique.

In this paper we present a genetic algorithm (GA) to solve the transmit power control problem in cognitive radio networks, formulating the optimization problem in terms of a general utility function. An admission control algorithm is used in conjunction with the GA to transform a non-feasible optimization problem in a feasible one for a subset of SUs. The GA is then used to solve the power allocation problem in terms of energy efficiency and the results are compared with a solution based on linear programming methods. Optimization methods based on linear or non-linear programming techniques in general allow obtaining better solutions than those found by heuristic optimization methods like GAs. However, exact optimization methods are not very flexible and generally require that objective function, its derivatives and constraints meet a set of continuity conditions. The proposed GA can be easily adapted to handle a variety of optimization objectives by only modifying the method employed to evaluate the fitness of each individual in the GA population. We demonstrate this by utilizing a fitness function that allows the joint optimization of total transmit power consumption and total capacity of the secondary network. Moreover, our results show a nearoptimum performance of the proposed GA aided TPC scheme based on the multi-objective fitness function.

The remainder of this paper is organized as follows. In Section II the system model and problem formulation are presented. Section III describes a solution to the TPC problem for energy efficiency while Section IV presents the proposed GA algorithm. In Section $\mathrm{V}$ numerical results are provided, while Section VI concludes the paper.

\section{SySTEM MODEL}

We consider an interference-limited spectrum sharing scenario in which a secondary network operates inside the primary coverage area. The primary and secondary networks are composed of $M$ and $N$ transmitter/receiver pairs, respectively. We assume that the signal propagation is dominated by path-loss effects. To model the link gain from the $k$ th transmitter to the $i$ th receiver, $h_{k i}$, we consider a log-distance path loss model, $h_{k i}=h_{0}\left(d_{0} / d_{k i}\right)^{\alpha}$, where $\alpha$ is the path loss exponent, $d_{k i}$ 
is the distance from the $k$ th transmitter to the $i$ th receiver, $d_{0}$ and $h_{0}$ are constants that represent the reference distance and the link gain at this location, respectively. It is also assumed that information about the locations of primary and secondary nodes is available for cognitive devices. Location-awareness can be achieved by using the positioning techniques in [8].

The employed QoS metric is the signal-to-interference-andnoise ratio (SINR), which at the $i$ th PU can be computed as

$$
\gamma^{(p)}=\frac{h_{i i}^{(p p)} p_{i}^{(p)}}{\sum_{j=1}^{N} h_{j i}^{(s p)} p_{j}^{(s)}+\sum_{\substack{j=1 \\ j \neq i}}^{M} h_{j i}^{(p p)} p_{j}^{(p)}+\sigma^{2}}, \quad i=1,2, \ldots, M,
$$

where $p_{i}^{(p)}$ and $p_{j}^{(s)}$ denote the transmit power of the $i$ th primary and the $j$ th secondary transmitters, respectively; $h_{j i}^{(s p)}$ and $h_{j i}^{(p p)}$ are the link gains from the $j$ th secondary and primary transmitters to the $i$ th primary receiver, respectively and $\sigma^{2}$ is the thermal noise power. Similarly, the SINR at the $i$ th SU is

$$
\gamma^{(s)}=\frac{h_{i i}^{(s s)} p_{i}^{(s)}}{\sum_{j=1}^{M} h_{j i}^{(p s)} p_{j}^{(p)}+\sum_{\substack{j=1 \\ j \neq i}}^{N} h_{j i}^{(s s)} p_{j}^{(s)}+\sigma^{2}}, \quad i=1,2, \ldots, N,
$$

where $h_{j i}^{(s s)}$ and $h_{j i}^{(p s)}$ are the link gains from the $j$ th secondary and primary transmitters to the $i$ th secondary receiver.

The SUs may only transmit if they satisfy the QoS requirements for both primary and secondary receivers. Primary transmitters employ fixed transmit powers. The secondary network is expected to limit the interference caused to the primary network while attempting to provide service to the SUs with a specified QoS determined by the target SINR. Therefore, the $i$ th link is considered active if the received SINR is above the corresponding threshold $\gamma_{i}^{(t h p)}$ for PUs and $\gamma_{i}^{(t h s)}$ for SUs. In order to meet these requirements, each SU can adjust its transmit power within the range $\left[p_{\min }, p_{\max }\right]$ and then a power control vector $\mathbf{p}=\left[p_{1}, \ldots, p_{N}\right]$, can be allocated to the secondary network. If the optimization goal is to minimize an objective function $\varphi(\mathbf{p})$, then the optimal power control vector $\mathbf{p}^{*}$ can be found by solving

$$
\begin{array}{cl}
\mathbf{p}^{*}=\underset{\mathbf{p}}{\arg \min } \varphi(\mathbf{p}) & \\
\text { subject to } & C_{1}: \gamma_{i} \geq \gamma_{i}^{(t h p)}, \quad i=1,2, \ldots, M \\
& C_{2}: \gamma_{i} \geq \gamma_{i}^{(t h s)}, \quad i=1,2, \ldots, N \\
& C_{3}: p_{\min } \leq p_{i} \leq p_{\max }, \quad i=1,2, \ldots, N .
\end{array}
$$

The problem is said to be feasible when there is at least one solution that satisfies all constraints. This global optimization approach requires that a central controller in the secondary network assigns the power vector to SUs by finding a solution to (3). A control channel between the central controller and the SUs would be necessary [9], [10].

\section{ENERGY EFFICIENCY OPTIMIZATION PROBLEM Solution By Linear Programming Method}

In [11], a solution to the problem stated in (3) is presented when the optimization goal is to minimize the total secondary network power consumption. In this case, the objective function is a linear function of the secondary network power vector, $\varphi_{e}(\mathbf{p})=\sum_{i=1}^{N} p_{i}^{(s)}$. The problem constraints can also be transformed into linear constraints, so that the problem can be solved by linear programming methods. Constraints $C_{2}$ in (3) can be represented as $[\mathbf{I}-\boldsymbol{\Gamma} \mathbf{A}] \mathbf{p} \leq \mathbf{b}$, where $\mathbf{I}$ is the $N \times N$ identity matrix, $\boldsymbol{\Gamma}$ is an $N \times N$ matrix whose elements are

$$
\gamma_{i j}=\left\{\begin{array}{ll}
\gamma_{i}^{(t h s)}, & i=j \\
0, & \text { otherwise }
\end{array},\right.
$$

A is an $N \times N$ matrix with entries specified as

$$
a_{i j}=\left\{\begin{array}{ll}
0, & i=j \\
h_{j i}^{(s s)} / h_{i i}^{(s s)}, & \text { otherwise }
\end{array},\right.
$$

and $\mathbf{b}$ is a row vector with elements

$$
b_{i}=\gamma_{i}^{(t h s)}\left[\sigma^{2}+\sum_{j=1}^{M} h_{j i}^{(p s)} p_{j}^{(p)}\right] / h_{i i}^{(s s)} \text {. }
$$

When $[\mathbf{I}-\boldsymbol{\Gamma} \mathbf{A}]^{-1}$ exists, and the power vector $\mathbf{p}^{*}=$ $[\mathbf{I}-\boldsymbol{\Gamma} \mathbf{A}]^{-1} \mathbf{b}$ satisfies constraints $C_{1}$ and $C_{3}$, the problem is feasible and the optimal solution is $\mathbf{p}^{*}$.

\section{A. Admission Control}

When the problem is not feasible, the initial optimization problem can be redefined by excluding a set of secondary links in a way that the problem becomes feasible for the remaining SUs. To accomplish this, an admission control algorithm is presented in [11], and for which we include the pseudo code in Algorithm 1. The method iteratively discards those users that caused the worst interference until the problem becomes feasible for a subset $S_{a} \subseteq S$, being $S$ the set of all SUs. The user that caused the worst interference in the secondary network can be determined by finding the index, $w$, of the highest value element in a vector $\mathbf{z}$ whose entries are

$$
z_{j}=\sum_{\forall i \in S_{a}}\left(a_{i j}+a_{j i}\right)
$$

This algorithm is independent of the objective function, and thus it can be used as a general way to guarantee that the problem to be solved is feasible.

\section{Genetic Algorithm ImplementATion}

\section{A. General Description}

A GA repeatedly modifies a population of solutions, also called individuals or chromosomes, which represent the search space. Over successive generations, a random initial population evolves until certain stopping criteria are met. As shown in Algorithm 2, at each iteration the GA selects the best individuals from the current population and uses them to produce the children for the next generation. The produced children are used to replace a fraction of the current population in the next generation. Children are obtained by means of 


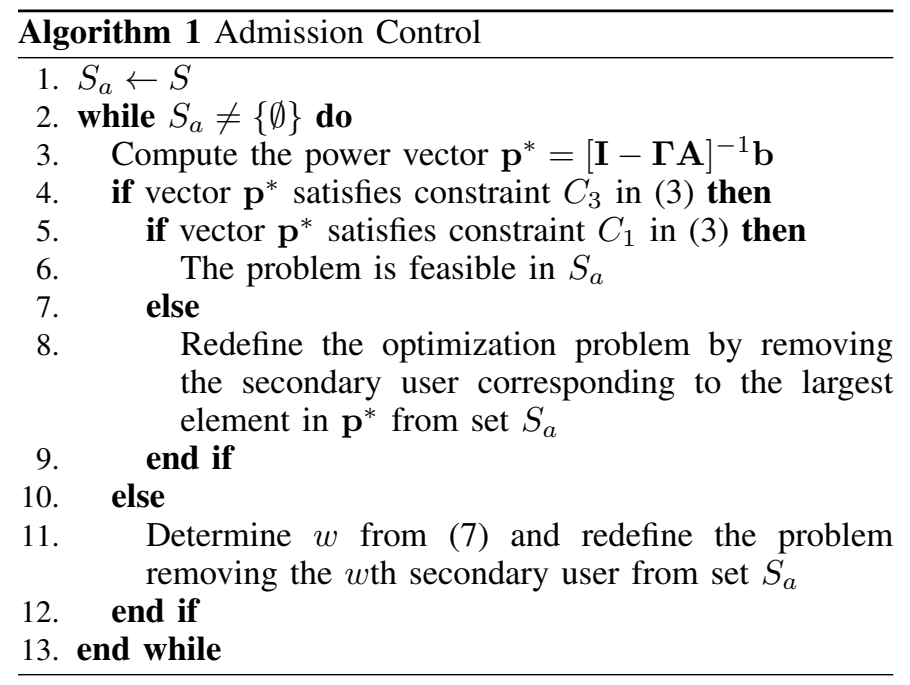

two processes: mutation and crossover. The crossover of two parent chromosomes tries to exploit the best genes of the previous generation to create a better offspring. The mutation can prevent the GA from getting stuck in a local maximum by randomly introducing little changes [12]. At each generation there is a group of chromosomes, called the elite individuals, which are guaranteed to survive to the next generation without modification. Denoting $\lambda_{e}, \lambda_{m}$ and $\lambda_{c}$ as the number of elite, mutation and crossover individuals in the population, respectively, the population size is given by $\lambda=\lambda_{e}+\lambda_{m}+\lambda_{c}$. Setting $\lambda$ and $\lambda_{e}$ to fixed values, the number of crossover and mutation children can be controlled via the crossover fraction parameter, defined as $\kappa=\lambda_{c} /\left(\lambda_{c}+\lambda_{m}\right)$.

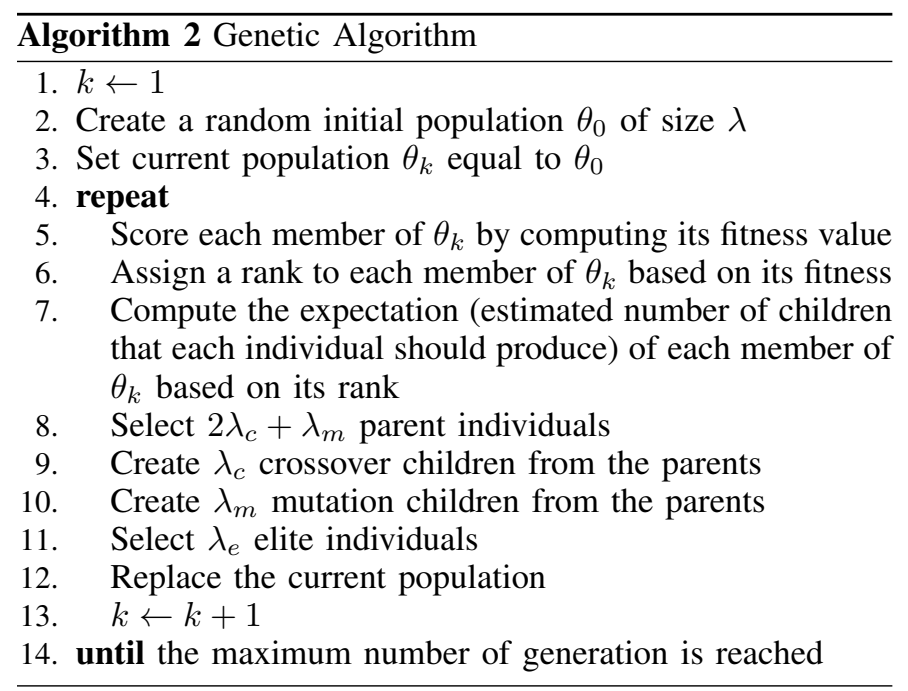

The general pseudocode shown in Algorithm 2 can be adapted to solve a variety of optimization problems, by only modifying the way each individual is scored and ranked. The optimization problem we are trying to solve via a GA is a constrained optimization problem. Hence, the fitness evaluation of each individual must take into account not only the objective function value for that individual, but also the constraints violations. For that sake, the admission control algorithm discussed in Section III is executed before the GA to ensure problem feasibility. Since the GA does not guarantee the optimality of the solution, when the algorithm stops it is checked if the power vector to be assigned to the secondary network does not violate the primary network SINR requirements, otherwise a null power vector is returned.

In order to evaluate the fitness of individuals we propose two functions. The first employs a penalty function to handle constraints and is oriented towards minimizing the total transmit power consumption of the secondary network. The second is a multi-objective fitness function that jointly optimizes the total capacity and transmit power consumption.

\section{B. Power Consumption Minimization}

The most common method to handle constraints in GAs is to use penalty functions [13]. Penalty methods are procedures for approximating constrained optimization problems by unconstrained ones. This is accomplished by adding to the objective function a term that prescribes a high cost for violation of the constraints. In this case the fitness value of each individual is computed as

$$
\phi=\varphi_{e}(\mathbf{p})+c_{p} \sum_{i=1}^{M} \max \left[0, g_{i}^{(p)}\right]^{2}+c_{s} \sum_{i=1}^{N} \max \left[0, g_{i}^{(s)}\right]^{2},
$$

where $g_{i}^{(p)}=\gamma_{i}^{(t h p)}-\gamma_{i}^{(p)}$ and $g_{i}^{(s)}=\gamma_{j}^{(t h s)}-\gamma_{j}^{(s)}$. The highest priority of PUs can be controlled by separating the primary and secondary constraints in the penalty function and assigning a higher value to the primary penalty factor $c_{p}$ than to the secondary penalty factor $c_{s}$.

\section{Joint Optimization of Power Consumption and Capacity}

In this case we use a rank based fitness scaling method, based on a multi-objective fitness function, which outputs a vector $\boldsymbol{\Phi}$ with four components

$$
\mathbf{\Phi}=\left[\sum_{i=1}^{M} \mu_{i}^{(p)}, \sum_{i=1}^{N} \mu_{i}^{(s)}, \varphi_{e}(\mathbf{p}), \varphi_{c}(\mathbf{p})\right] .
$$

The first and second entries count the number of primary and secondary network constraints violations, respectively:

$$
\begin{aligned}
& \mu_{i}^{(p)}=\left\{\begin{array}{ll}
1, & \gamma_{i}^{(p)}<\gamma_{i}^{(t h p)} \\
0, & \text { otherwise }
\end{array},\right. \\
& \mu_{i}^{(s)}=\left\{\begin{array}{ll}
1, & \gamma_{i}^{(s)}<\gamma_{i}^{(t h s)} \\
0, & \text { otherwise }
\end{array} .\right.
\end{aligned}
$$

The third and fourth entries account for the total transmit power and capacity of the secondary network, respectively. The function $\varphi_{c}(\mathbf{p})=-\sum_{i=1}^{N} \log _{2}\left[1+\gamma_{i}^{(s)}\right]$ denotes the objective function aimed at maximizing the total capacity of the secondary network which takes into account the sum of the capacity of the $N$ sub-links in a bandwidth normalized analysis, based on the Shannon-Hartley channel capacity theorem, as has been presented in [4], [7]. Note that the negative sign means that the optimization goal is to minimize the objective function, so that the smallest possible value of $\varphi_{c}(\mathbf{p})$ corresponds to the highest capacity of the secondary network.

When ranking two individuals $X$ and $Y$, the constraints entries are compared according to the following priority: 1) 
number of primary constraints violations and 2) number of secondary constraints violations. In each case, the next entry is considered for comparison only if the current entry is the same for both individuals. If they are different, the individual with the lowest entry value is selected as the best. Next, if the number of constraints violations is equal for both individual, a parameter $\Delta \varphi$ that accounts for the joint improvement in capacity and power consumption is computed

$$
\Delta \varphi=\delta_{e} \frac{\varphi_{e}^{(X)}-\varphi_{e}^{(Y)}}{\left|\varphi_{e}^{(X)}+\varphi_{e}^{(Y)}\right|}+\delta_{c} \frac{\varphi_{c}^{(X)}-\varphi_{c}^{(Y)}}{\left|\varphi_{c}^{(X)}+\varphi_{c}^{(Y)}\right|} .
$$

If $\Delta \varphi<0$ the chromosome $X$ is better than $Y$, else the individual $Y$ is selected. Parameters $\delta_{e}$ and $\delta_{c}$ control the tradeoff between power consumption minimization and total capacity maximization. If $\delta_{e}=1$ and $\delta_{c}=0$, then only the power consumption is optimized. If we set $\delta_{e}=0$ and $\delta_{c}=1$ then only the total capacity is maximized.

\section{Parents Selection}

To select the parents for the next generation it is necessary to map the raw fitness scores to an expected number of children for each individual. The selection process requires that the expected number of children be in a proper range [14]. Regarding the employed fitness scaling method, the expectation of each individual is computed as

$$
\epsilon_{j}=\frac{1 / \sqrt{q_{j}}}{\sum_{i=1}^{\lambda}\left(1 / \sqrt{q_{i}}\right)},
$$

where $q_{k}$ represents the rank of the $k$ th chromosome in the population. If we are minimizing, $q_{k}=1$ corresponds to the best chromosome while $q_{k}=\lambda$ corresponds to the worst. Note that the sum of all expectations must be equal to one.

The selection process chooses parents based on their expectation. The selection method used in our GA was the roulette method. The algorithm simulates a roulette wheel, where the area of each segment is proportional to its expectation and uses a random number to select one of the sections with a probability equal to its area. In this method each $j$ th individual from the current population has a probability equal to $\epsilon_{j}$ of being selected as parent for the next generation.

\section{E. Mutation and Crossover}

The mutation of each individual is performed by adding to the parent chromosome a random vector taken from a Gaussian distribution with zero mean. The variance of this distribution can be controlled via two parameters: the mutation scale, $M_{s}$, and the mutation shrink, $M_{h}$. The mutation scale determines the variance at the first generation. The mutation shrink controls how the variance shrinks as generations go by. At generation $g$, the mutation variance is computed as $M_{\sigma}=M_{s}\left(1-g M_{h} / S_{g}\right)$, where $S_{g}$ is the maximum number of generations. If $M_{h}=0$, the variance is constant and if $M_{h}=1$, the variance shrinks to 0 linearly as the last generation is reached. To produce a crossover child, once two parents are randomly selected, the genes in the offspring are chosen from one or another parent with equal probability.

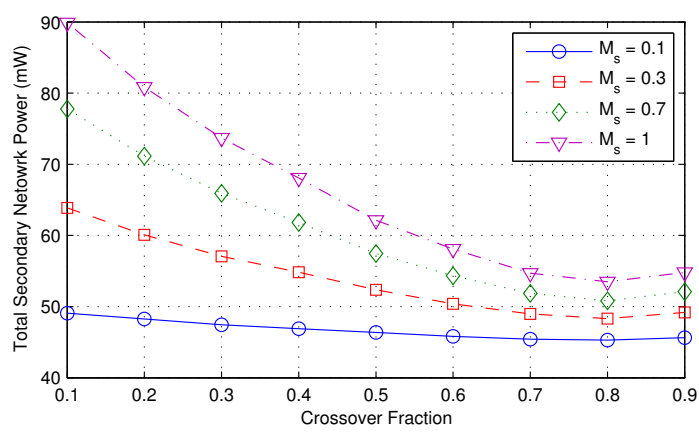

Fig. 1. Average value of total transmit power of secondary network as a function of the crossover fraction and for different values of the mutation scale, using the penalty-based fitness function and assuming $N=10, \lambda=50$ and $S_{g}=500$.

\section{NUMERICAL RESULTS}

In our simulations the topology is generated by the uniform random placement of $N$ cognitive transmitters in a cell of radius $R=100 \mathrm{~m}$. We consider the presence of a single primary link $(M=1)$, with the transmitter at the center of the cell. Each receiver is placed at a fixed distance $r=10 \mathrm{~m}$ from the intended transmitter in a uniformly generated random direction. For the propagation model we consider $\alpha=3$, $d_{0}=1 \mathrm{~m}, h_{0}=10^{-4}$. The SINR threshold was set to $10 \mathrm{~dB}$ and $3 \mathrm{~dB}$ for the primary and secondary networks, respectively. For all cognitive transmitters we set $p_{\min }=0 \mathrm{~mW}$ and $p_{\max }=$ $200 \mathrm{~mW}$, and for the primary transmitter $p^{(p)}=100 \mathrm{~mW}$.

\section{A. Tuning the GA Parameters}

First, we illustrate the results of adjusting the most important parameters of the GA for $N=10$ assuming that the optimization objective is to minimize the total secondary network transmission power, $P_{s}$. These parameters include the crossover fraction, the mutation scale, the population size and the maximum number of generations. We assume a number of elite individuals $\lambda_{e}=2$ and a mutation shrink $M_{h}=1$. Also, for the penalty-based fitness function we set penalty parameters to $c_{p}=8$ and $c_{s}=2$. For the multi-objective fitness function we set $\delta_{e}=1$ and $\delta_{c}=0$, since the goal is to minimize the total secondary network transmission power.

Figure 1 shows the average value of $P_{s}$ as a function of the crossover fraction and for different values of the mutation scale, using the penalty-based fitness function. Similar results were obtained by using the multi-objective fitness function. The crossover fraction controls the balance between the exploration of new solutions (mutation) and the recombination of genes from parents with the highest fitness of past generations (crossover). We can see that this balance is reached when $\kappa \approx 0.8$. Mutation adds to the diversity of a population and thereby increases the likelihood that the algorithm generates individuals with better fitness values. However, from Figure 1 it can be noted a degradation in performance when the mutation scale is increased or the crossover fraction is decreased, since the genetic search becomes a random search.

Figure 2 shows the average value of $P_{s}$ as a function of the population size using the penalty-based (GAp) and multiobjective (GAm) fitness functions. If the population size is too 


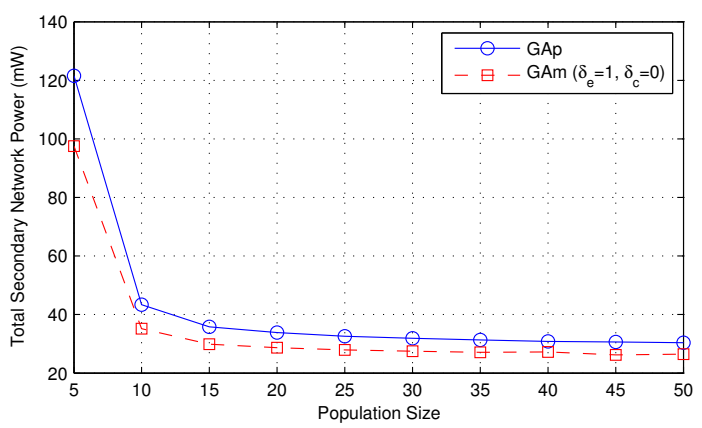

Fig. 2. Average value of total transmit power of secondary network as a function of the population size, assuming $N=10, \kappa=0.8, M_{s}=0.1$ and $S_{g}=100$.

small, the search space is poorly represented and the GA may converge prematurely. Larger population sizes allow the GA to explore more solutions from the search space and therefore a better result is obtained. However, the larger the population size, the longer the GA takes to compute each generation. An optimal selection of the population size allows to find the solution without a high consumption of computational resources and time. The optimum value of $\lambda$ is dependent on the genome length, as for individuals of a population to cover the entire search space, it is necessary that the size of the population be greater than the genome length. As a result, the GA performance does not increase linearly with the increasing of population size, so the improved performance is more remarkable at lower values of $\lambda$. In setting this parameter should be considered the relationship between the solution quality and the computational cost, which is directly proportional to $\lambda$. As shown in Figure 2, for a population size greater than 45 (about four times the genome length) there is not a significant improvement in the performance.

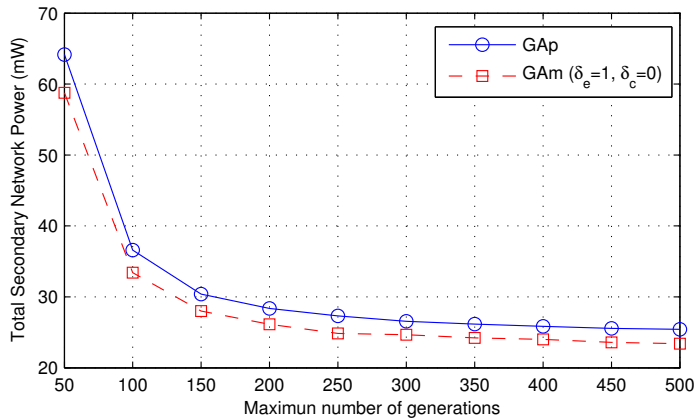

Fig. 3. Average value of total transmit power of secondary network as a function of the maximum number of generations, assuming $N=10, \kappa=0.8$, $M_{s}=0.1$ and $\lambda=50$

Figure 3 shows the average value of $P_{s}$ as a function of the maximum number of generations. In our GA implementation, a maximum number of iterations is used as stopping criteria. Generally, the progress of a GA is most noticeable during the first generations, but after a certain number of iterations this progress becomes slower. Although the convergence time is very important, a rapid convergence is not always desirable, because if the fitness function has a large number of local optima, the algorithm may converge to one of these local optima. In these cases, the number of generations and the population diversity required by the GA may be considerably higher than in cases where the objective function has fewer local maxima. Taking into account the results shown in Figure 3 , we select $S_{g}=500$ as an appropriate value.

\section{B. Minimizing Total Transmit Power}

Figure 4 shows the average value of $P_{s}$ as a function of the number of SUs. In this figure the results obtained via linear programming (LP) and the GA using the penalty-based and multi-objective fitness functions are compared. Note how the performance of the proposed GA scheme is very close to the exact solution. However, since an admission control algorithm is used, these results have to be analyzed with care. We must take into account the probability of SINR constraint violation at secondary receivers (outage probability). This outage probability is shown in Figure 5 as a function of the number of secondary links. These results demonstrate how, regardless of the results shown in Figure 4, the penaltybased fitness function (GAp) has a worse performance when compared to the multi-objective fitness function (GAm) for this particular problem. Moreover, note that the outage probability that is achieved with the GAm is slightly greater than the one obtained with the LP, what means that even if the admission control algorithm ensures optimality, still exists a non-zero probability that the GA rejects users from feasible set $S_{a}$ in favor of minimizing the objective function. Thus, the fact that the GAm outperforms the LP solution in Figure 4 comes at the cost of an increased outage probability.

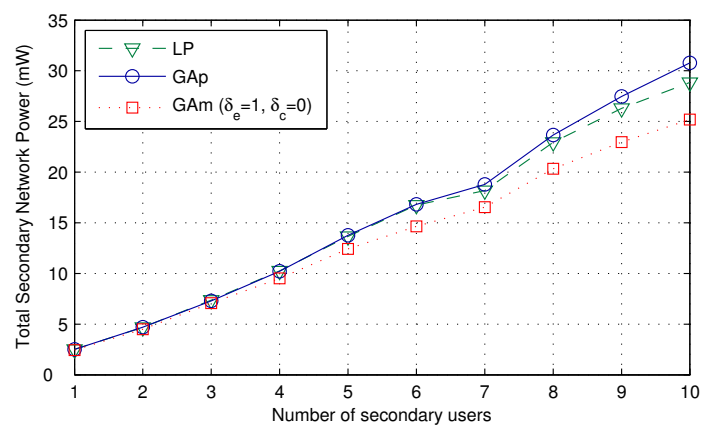

Fig. 4. Average value of total transmit power of secondary network as a function of the number of secondary users.

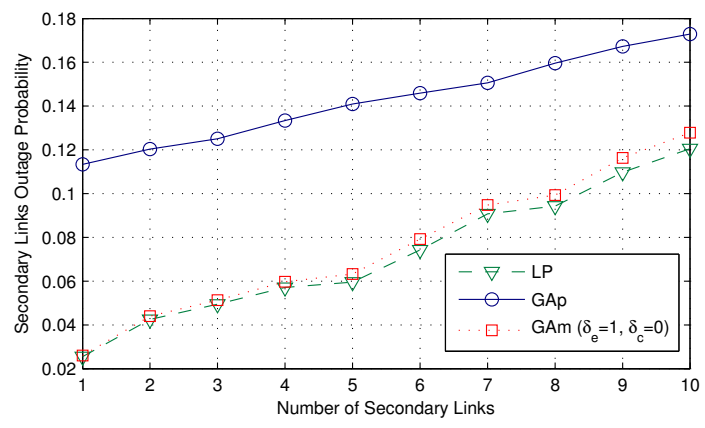

Fig. 5. Outage probability as a function of the number of secondary users.

\section{Joint Optimization of Capacity and Transmit Power}

Figure 6 shows the improvement in the secondary network capacity that is achieved using the multi-objective fitness func- 


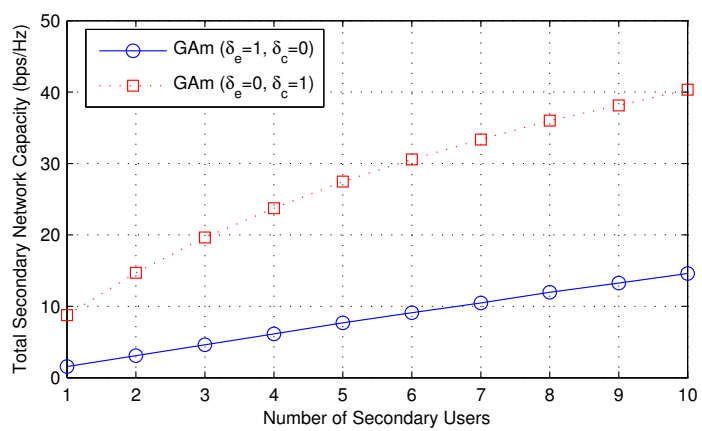

Fig. 6. Average value of total capacity of secondary network as a function of the number of secondary users.

tion with parameters $\delta_{e}=0$ and $\delta_{c}=1$ (capacity maximization), when compared with this same fitness function but using parameters values $\delta_{e}=1$ and $\delta_{c}=0$ (power minimization). However, as can be noted in Figure 7, this improvement prescribes a high cost in the power consumption. An increase in network capacity on the order of 2.5 times requires that the power consumption is increased by approximately 40 times. Figure 8 shows how by setting $\delta_{e}=1$ and varying $\delta_{c}$ between 0 and 20 the tradeoff between power minimization and capacity maximization can be controlled. Thus, the secondary network power consumption can be adjusted as a function of the throughput requirements and vice versa.

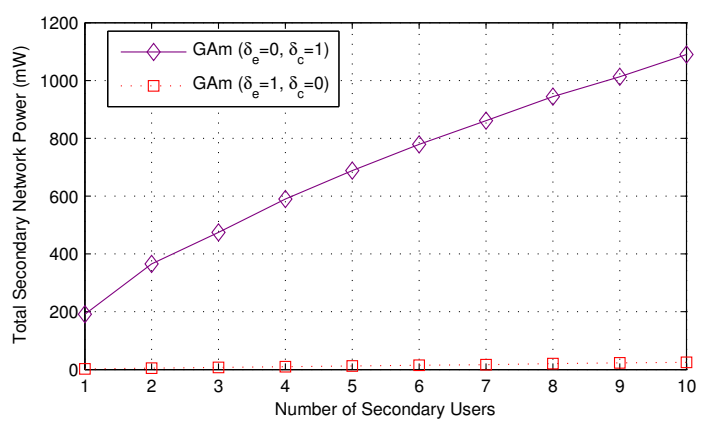

Fig. 7. Average value of total transmit power of secondary network as a function of the number of secondary users.

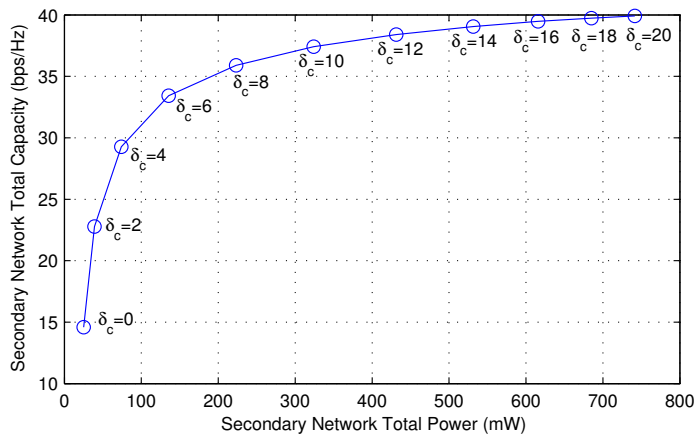

Fig. 8. Tradeoff between power consumption minimization and total capacity maximization, for $N=10$.

\section{CONCLUSION}

The use of a GA is an attractive approach to solve optimization problems in cognitive radio networks. The main limitation found with the use of a GA to solve the optimization problem under study was that multiple parameters must be adjusted in order to ensure the proper operation of the algorithm. By using a multi-objective fitness function, the algorithm implementation can be easily adapted to solve a variety of optimization problems, regardless of the number and type of constraints. The use of a penalty function to handle the constraints does not guarantee the strict fulfillment of all constraints and additional parameters should be introduced which must also be adjusted to achieve a tradeoff between maximizing the utility and meting the constraints. In future works we will investigate how to increase the algorithm flexibility by dynamically adjusting its parameters and combining multiple optimization objectives. As future work, we will focus on full-duplex cognitive networks.

\section{ACKNOWLEDGMENT}

Authors would like to thank the Infotech Oulu Graduate School, University of Oulu, Academy of Finland and Tekes. This paper was also supported by CNPq and Capes (Brazil).

\section{REFERENCES}

[1] B. Wang and K. J. R. Liu, "Advances in Cognitive Radio Networks: A Survey," IEEE Journal of Selected Topics in Signal Processing, vol. 5, pp. 5 - 22, 2011.

[2] Y.-C. Liang, K.-C. Chen, G. Y. Li, and P. Mhnen, "Cognitive Radio: Networking and Communications An Overview," IEEE Transactions on Vehicular Technology, vol. 60, pp. 3386 - 3407, 2011.

[3] S. Buzzi and D. Saturnino, "A Game-Theoretic Approach to EnergyEfficient Power Control and Receiver Design in Cognitive CDMA Wireless Networks," IEEE Journal of Selected Topics in Signal Processing, vol. 5, pp. 137-150, Feb. 2011.

[4] S. M. Sanchez, R. D. Souza, E. M. G. Fernandez, and V. A. Reguera, "Rate and Energy Efficient Power Control in a Cognitive Radio Ad Hoc Network," IEEE Signal Processing Letters, vol. 20, pp. 451-454, 2013.

[5] S. Parsaeefard and A. R. Sharafat, "Robust Distributed Power Control in Cognitive Radio Networks," IEEE Journals \& Magazines, vol. 12, pp. 609-620, 2013.

[6] M.-L. Ku, L.-C. Wang, and Y.-T. Su, "Optimal Multiuser Beamforming and Power Allocation for Hierarchical Cognitive Radio Systems," in Proc. International Symposium on Information Theory and its Applications (ISITA), 2010, pp. 946-951.

[7] S. M. Sanchez, R. D. Souza, E. M. G. Fernandez, and V. A. Reguera, "Impact of Power Allocation and Antenna Directivity in the Capacity of a Multiuser Cognitive Ad Hoc Network," Radioengineering, vol. 21, pp. 1110-1116, Dec. 2012.

[8] H. Celebi, I. Guvenc, S. Gezici, and H. Arslan, "Cognitive-Radio Systems for Spectrum, Location, and Environmental Awareness," IEEE Ant. and Prop. Mag., vol. 52, pp. 41-61, 2010.

[9] L. Lu, X. Zhou, U. Onunkwo, and G. Y. Li, "Ten years of research in spectrum sensing and sharing in cognitive radio," EURASIP Journal on Wireless Communications and Networking, pp. 1 -16, 2012.

[10] A. D. Domenico, E. C. Strinati, and M.-G. D. Benedetto, "A Survey on MAC Strategies for Cognitive Radio Networks," IEEE Comm. Surveys \& Tutorials, vol. 14, 2012.

[11] L. Qian, X. Li, J. Attia, and Z. Gajic, "Power Control for Cognitive Radio Ad Hoc Networks," in Proc. 15th IEEE Workshop on Local an Metropolitan Area Networks, 2007, pp. 7-12.

[12] J. Brownlee, Clever Algorithms: Nature-Inspired Programming Recipes. Sidney, Australia: LuLu, 2011.

[13] Ö. Yeniay, "Penalty function methods for constrained optimization with genetic algorithms," Mathematical and Computational Applications, vol. 10, pp. 45-56, 2005.

[14] S. Ventura, Genetic programming: New approaches and successful applications Rijeka, Croatia: InTech, 2012. 